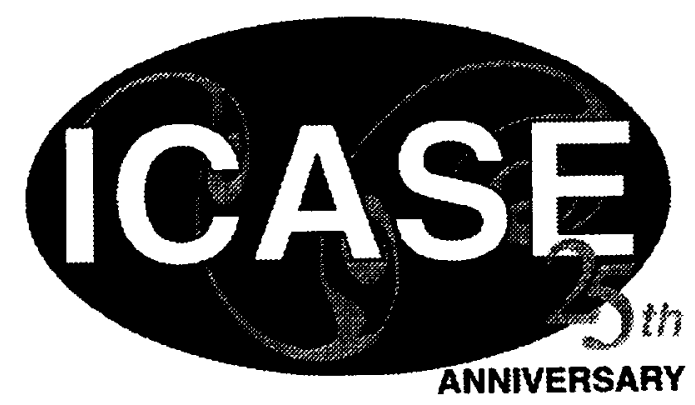

\title{
NUMERICAL SIMULATION OF TURBULENT JETS WITH RECTANGULAR CROSS-SECTION
}

\section{Robert V. Wilson Ayodeji O. Demuren}

NASA Contract No. NAS1-19480

January 1997

Institute for Computer Applications in Science and Engineering NASA Langley Research Center Hampton, VA 23681-0001

Operated by Universities Space Research Association

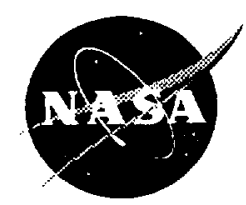

National Aeronautics and Space Administration

Langley Research Center Hampton, Virginia 23681-0001 



\title{
NUMERICAL SIMULATION OF TURBULENT JETS WITH RECTANGULAR CROSS-SECTION ${ }^{*}$
}

\author{
Robert V. Wilson and Ayodeji O. Demuren \\ Department of Mechanical Engineering \\ Old Dominion University \\ Norfolk, Virginia 23529, USA
}

\begin{abstract}
Three-dimensional turbulent jets with rectangular cross-section are simulated with a finite-difference numerical method. The full Navier-Stokes equations are solved at low Reynolds numbers, whereas at the high Reynolds numbers filtered forms of the equations are solved along with a subgrid scale model to approximate effects of the unresolved scales. A 2-N storage, third-order Runge-Kutta scheme is used for temporal discretization and a fourth-order compact scheme is used for spatial discretization. Computations are performed for different inlet conditions which represent different types of jet forcing. The phenomenon of axis- switching is observed, and it is confirmed that this is based on self-induction of the vorticity field. Budgets of the mean streamwise velocity show that convection is balanced by gradients of the Reynolds stresses and the pressure.
\end{abstract}

\footnotetext{
. "The authors were partially supported by the National Aeronautics and Space Administration under NASA Contract No. NAS 1-19480 while the authors were in residence at the Institute for Computer Applications in Science and Engineering (ICASE), NASA Langley Research Center, Hampton, VA 23681-0001. Additional support was provided by the NASA Graduate Student Research Program.
} 



\section{INTRODUCTION}

Turbulent jets are present in many physical processes and technological applications. Turbulent jets can be found in combustors where the fuel and oxidizer are introduced as co-flowing jets. The efficiency of such a process is largely determined by the mixing of the jets. Recently, jet aircraft noise has received much attention due to plans for a high-speed civil transport vehicle. A critical issue for the project's success is reducing jet noise to acceptable levels near populated areas. The belief is that acoustic patterns can be altered by manipulating the large scale structures in turbulent jet flows through external forcing. Non-circular jets can also be used to enhance the mixing of hot jet gases with the surroundings and thus avoid aircraft detection. In industrial applications, efficient mixing is required to dilute pollutants issuing from smokestacks with the ambient air to minimize its harmful effects or to promote fuel-oxidant mixing in combustion chambers.

Experiments (Tsuchiya et al. 1986, 1989, Quinn 1989, 1994) have shown that three-dimensional (3-D) jets can be used to enhance mixing and entrainment rates in comparison to nominally two-dimensional (2-D) jets. A fundamental understanding of the dynamics of complex, turbulent jets is required for their prediction and control. The present study is concerned with the understanding of the role of vorticity in the spatial evolution of incompressible 3-D jets in the near to medium field and the effects of external forcing thereupon. Previous numerical simulations of such flows have been performed by Grinstein (1995), with emphasis on the vorticity dynamics in the near field (less than 5 diameters), and Miller et al. (1995), who studied co-flowing jets at a low Reynolds number of 800 . In the latter, examination of intantaneous vorticity fields show symmetries which are not indicative of turbulent flow. This work extends those studies into the medium field, beyond the extent of the potential core, and into truly turbulent flows.

\section{MATHEMATICAL FORMULATION}

The partial differential equations governing the incompressible jet fluid flow are the NavierStokes equations which can be written in Cartesian tensor form, for dimensionless variables as:

$$
\frac{\partial u_{i}}{\partial t}+u_{j} \frac{\partial u_{i}}{\partial x_{j}}=-\frac{\partial p}{\partial x_{i}}+\frac{1}{R e_{D_{e}}} \frac{\partial^{2} u_{i}}{\partial x_{j} \partial x_{j}}
$$


where, $u_{i}$ are the Cartesian velocity components in the Cartesian coordinate directions $x_{i}, p$ is the pressure and $\operatorname{Re}_{\mathrm{D}^{*}}$ is the Reynolds number based on the equivalent diameter. These equations must be solved in conjuction with the continuity equation:

$$
\frac{\partial u_{i}}{\partial x_{i}}=0
$$

which expresses the divergence-free velocity condition.

At higher Reynolds numbers, all scales present in the flow cannot be resolved on computational grids that present resources would allow, so a large eddy simulation (LES) must be utilized. The application of a grid filter, $\bar{G}$, to equations (1) and (2) results in the filtered equations of motion, in which the overbar indicates a filtered variable:

$$
\frac{\partial \bar{u}_{i}}{\partial t}+\bar{u}_{j} \frac{\partial \bar{u}_{i}}{\partial x_{j}}=-\frac{\partial}{\partial x_{i}} \bar{p}+\frac{1}{R e_{D_{e}}} \frac{\partial^{2} \bar{u}_{i}}{\partial x_{j} \partial x_{j}}-\frac{\partial \tau_{i j}}{\partial x_{j}}
$$

and

$$
\frac{\partial \bar{u}_{i}}{\partial x_{i}}=0
$$

where $\bar{u}_{i}$ represents components of the filtered velocity which is resolved in the computation, and $\tau_{i j}=\rho \bar{u}_{i} u_{j}-\rho \bar{u}_{i} \bar{u}_{j}$ is the subgrid scale (SGS) Reynolds stress which must be modeled in terms of the resolved velocity field. The Smagorinsky eddy-viscosity model is utilized in the present study to approximate the SGS Reynolds stress as:

$$
\tau_{i j}-\frac{\delta_{i j}}{3} \tau_{k k}=-2 C_{s} \bar{\Delta}^{2}\left|\bar{S}_{i j}\right| S_{i j}
$$

where $\bar{\Delta}$ is a length scale associated with the grid size, $\bar{S}_{i j}=\frac{1}{2}\left(\frac{\partial \bar{u}_{i}}{\partial x_{j}}+\frac{\partial \bar{u}_{j}}{\partial x_{i}}\right)$ is the resolved strain rate tensor, and $\left|\bar{S}_{i j}\right|=\sqrt{2 \bar{S}_{i j} \bar{S}_{i j}}$. The model coefficient, $C_{s}$, is set to the constant value of 0.01 . 
The Navier-Stokes equations (1) and the filtered equations (3) are discretized temporally with explicit Runge-Kutta (RK) schemes and spatially with implicit compact finite difference schemes. The discretized equation has the form:

$$
u_{i}^{n+1}=u_{i}^{n}+b^{M} \Delta t\left[H_{i}^{M}-\delta_{x_{i}} P^{M}\right]
$$

with

$$
H_{i}^{M}=-u_{j} \delta_{x_{j}} u_{i}^{M}+\frac{1}{R e_{D_{e}}} \delta_{x x_{j}} u_{i}^{M}
$$

i.e., the sum of convection and diffusion $(\mathrm{CD})$ terms.

In Eq.(6), $n$ represents the time step and $M$ stands for the $M_{t h}$ stage of the RK scheme, with the corresponding coefficient $b^{M} . \delta_{x_{j}}$ and $\delta_{x x_{j}}$ are compact first and second derivative operators, respectively. In the present study low-storage RK schemes are utilized. The low-storage requirement is accomplished by continuously overwriting the storage location for the time derivatives and unknown variables at each sub-stage:

$$
\begin{gathered}
\hat{H}_{i}^{M} \leftarrow a^{M} \hat{H}_{i}^{M} \\
u_{i}^{M+1} \leftarrow u_{i}^{M}+b^{M} \Delta t \hat{H}_{i}^{M}
\end{gathered}
$$

where $\hat{H}_{i}^{M}=H_{i}^{M}-\left[\partial p^{M} / \partial x_{i}\right]$ and the notation $\leftarrow$ is used to indicate that the storage locations, $\hat{H}_{i}^{M-1}, u_{i}^{M}$ are overwritten by, $\hat{H}_{i}^{M}, u_{i}^{M+1}$, respectively. The coefficients $a^{M}$ and $b^{M}$ for the low-storage, third--order scheme (Williamson, 1980; Lowery and Reynolds 1986) are,

Table 1: Coefficients of the third-order RK scheme

\begin{tabular}{|c|c|c|}
\hline$M$ & $a^{M}$ & $b^{M}$ \\
\hline \hline 1 & 0 & 0.500 \\
\hline 2 & -0.68301270 & 0.91068360 \\
\hline 3 & -1.33333333 & 0.36602540 \\
\hline
\end{tabular}


Compact finite-difference schemes (Lele 1992) used to approximate derivatives which appear in Eqs. (1 - 5) involve implicit treatment of discrete derivatives and explicit treatment of discrete functions. Only tri-diagonal schemes are utilized in this study. For example:

$$
\alpha \phi_{i-1}^{\prime}+\phi_{i}^{\prime}+\alpha \phi_{i+1}^{\prime}=\frac{a}{2 \Delta x_{i}}\left(\phi_{i+1}-\phi_{i-1}\right)
$$

where $\phi_{i}^{\prime}$ represents the first derivative of the generic variable $\phi$ with respect to $x_{i}$, and $\alpha$, are the coefficients of the compact scheme which determine the accuracy. For the fourth-order scheme: $\alpha=1 / 4 ; a=3 / 2$. The LHS of Eq. (9) contains the unknown derivatives at grid points $i$ and $i \pm 1$ while the RHS contains the known functional values $\phi_{i}$ at the grid points $i \pm 1$. Similarly, the second derivative terms present in the viscous terms of the momentum equation and the Laplacian operator of the Poisson equation for pressure are approximated using fourth-order compact finite differences:

$$
\alpha \phi^{\prime \prime}{ }_{i-1}+\phi_{i}+\alpha \phi^{\prime \prime}{ }_{i+1}=\frac{a}{\left(\Delta x_{i}\right)^{2}}\left(\phi_{i+1}+2 \phi_{i}+\phi_{i-1}\right)
$$

where $\phi{ }_{i}{ }_{i}$ represents the second derivative of the generic variable $\phi_{i}$ with respect to $x_{i}$, and $\alpha$, a, $\mathrm{b}$ are the coefficients of the compact scheme. For the fourth-order scheme: $\alpha=1 / 10$; $a=6 / 5$. The tri-diagonal system of algebraic equations are solved using the Thomas algorithm. At the boundary third-order. one-sided differences are used to close the system of equations arising from the first or second derivative schemes.

A major requirement in the computation of incompressible flow is the satisfaction of the divergence-free velocity condition. This is made particularly difficult by the absence of an evolution equation for pressure. This condition must be satisfied indirectly through the solution of a Poisson equation derived by taking the divergence of the momentum equations:

$$
\nabla^{2} P^{M}=\delta_{x_{1}}\left[H_{i}^{M}+\frac{u_{i}^{M}}{b^{M} \Delta t}\right]
$$

The discretization of the Laplacian operator $\nabla^{2}$ results in: 


$$
\delta_{x x_{i}} P^{M}=\delta_{x_{i}}\left[H_{i}^{M}+\frac{u_{i}^{M}}{b^{M} \Delta t}\right]
$$

It is necessary to solve this equation completely at every substage of the RK scheme. Although the computational matrix is sparse, direct methods of solutions are not feasible. An iterative multigrid method was found to be most efficient, with reasonably good convergence rates. Nevertheless, the solution of the Poisson equation accounts for about $50 \%$ of the total computational effort.

In order to solve a well posed problem, the boundary and initial conditions for the jet simulations are defined. The ellipticity in the spatial terms of the governing equations requires that boundary conditions be defined on all boundaries. In the laboratory, jet flows are commonly generated by the use of a fan which forces fluid along an enclosed duct of nozzle. The jet leaves the exit plane of the nozzle where it interacts with the ambient fluid. Prior to exit, the jet can be considered as a relatively uniform freestream and a curved boundary layer at the walls of the nozzle. A short distance downstream of the nozzle exit, the boundary layer is smoothened so that the streamwise velocity can be modeled using the hyperbolic tangent (tanh) function. The tanh function enables the streamwise velocity to transition in the radial direction from the uniform velocity at the core of the jet to that of the ambient fluid in the freestream. The inflow boundary of the computational domain is placed at a short distance downstream of the nozzle exit which is not actually included in the jet simulations.

The mean or time-averaged streamwise velocity component at the inflow boundary is given by:

$$
\overline{u_{1}}\left(0, x_{2}, x_{3}\right)=U_{c}+\frac{\left(U_{H}-U_{L}\right)}{2} \tanh \left(\frac{r}{2 \theta_{o}}\right)
$$

where $U_{C}=\left(U_{H}+U_{L}\right) / 2$ is the convective velocity and $U_{H}, U_{L}$, represent the velocities of the jet core, and ambient fluid, respectively. The variable $r$, represents the minimum directed distance from the point, $\left(0, x_{2}, x_{3}\right)$ to the line of constant convective velocity of the boundary layer. The momentum thickness of the boundary layer at the inflow plane, $\theta_{o}$ is used to normalize the directed distance, $r$. If the point $\left(0, x_{2}, x_{3}\right)$ is "outside" the boundary layer, $r$ is defined to be nega- 
tive, otherwise $r$ is defined to be positive in the point lies on the inside of the boundary layer. Equation (13) produces a constant thickness boundary layer if the momentum thickness, $\theta_{o}$, is constant at all azimuthal positions along the boundary layer. Non constant thickness boundary layers can be generated by specifying the desired variation of $\theta_{o}$ along the perimeter of the boundary layer. The mean major and minor direction velocity components at the inflow plane, $u_{2}\left(0, x_{2}, x_{3}\right)$ and $u_{3}\left(0, x_{2}, x_{3}\right)$ are specified from experiment.

To simulate instabilities in the boundary layer, a time dependent forcing function of low intensity is added to the mean velocity components at the inflow boundary to promote unsteady motion. At higher Reynolds numbers or large computational lengths small round-off errors would grow to produce unsteady motion of the unstable shear layers thus obviating the need for forcing functions. Two classes of perturbations are used in the current study; (i) sinusoidal perturbations with frequency and amplitudes related to the most unstable modes found from viscous stability analysis (Wilson and Demuren 1996); (ii) perturbations having an experimentally measured velocity spectrum and transverse root mean square ( $r m s)$ value. Perturbations which have a broad spectrum resembling that of fully-developed, mostly random, 3-D turbulence were generated (Wilson 1993). The perturbations are typical of those found in the experimental jets originating from contoured nozzles. The power spectrum and rms values are taken from experiment. Because phase information is not included in the power spectrum, a random phase relationship for the modes comprising the spectrum was assumed. The random inlet boundary conditions are the spatial analog to the random perturbations generated for initial conditions in temporal simulations. Class (i) represents jets issuing from a nozzle with laminar boundary layers, whereas class (ii) represents jets issuing from a nozzle with turbulent boundary layers.

\section{MODEL PROBLEMS}

Spatial simulations of rectangular jet flows are performed in this study in which a fixed region of the flow is computed and disturbances grow in the streamwise direction. This can be contrasted with a temporal simulation in which a small region of the flow is followed in time and the domain moves in the streamwise direction. Spatial simulations are closer to reality, but are computation-

ally more demanding. As a result of the spatial reference frame, initial conditions are of minor 
importance because they are quickly convected out of the domain and the dynamics of the jet flow are determined by the forcing functions applied at the inflow plane, until the inherent instabilities of the flow take over or the onset of transition to turbulent flow. Simulations are started on coarser grids with the velocities specified at the inflow plane used to initialize the velocity field in the interior at $t=0$. After several flow through times (the time required for a fluid particle to convect from the inflow to the outflow plane at the jet core velocity, $U_{e}$ ) the initial conditions are "washed" from the domain. Simulations on finer grids are commenced with initial fields obtained by prolongating the coarser grid results using a standard, 2nd-order accurate interpolation formula.

The rectangular jet of the present study has a nominal aspect ratio of $2: 1$. This corresponds to the configuration of some jet issuing from contoured nozzle studied by Quinn (private communication). Figure 1(c) illustrates the shape of the jet cross-section at exit. Cases with both low and high Reynolds numbers are considered. In the former, the flow is fully resolved and no sub-grid scale model is required, but in the latter, filtered equations are solved and an SGS model is utilized. Reynolds numbers based on the core velocity and the equivalent diameter are 750 and 75000 , respectively. The four cases presented in this paper correspond to the two Reynolds numbers and the different types of inlet forcing functions, as explained previously, namely; (i) sinusoidal perturbations with the fundamental mode at an $r m s$ of $3 \%$ of mean jet velocity, and $\operatorname{Re}=750$; (ii) sinusoidal perturbation with the fundamental and first subharmonic modes, each at an $r m s$ of $1.5 \%$, and $\operatorname{Re}=750$; (iii) same perturbations as in (ii), but at $\operatorname{Re}=75000$; (iv) random broad-mode perturbations with an $r m s$ of $3 \%$ of mean jet velocity, and $\operatorname{Re}=75000$. Case (i) was simulated in a domain with size $(10 \times 5 \times 5)$ on a uniform computational grid of $(80 \times 65 \times 65)$, whereas all other cases were simulated on a $(10 \times 10 \times 10)$ domain with computational grids of $(100 \times 129 \times 129)$. The computational grids correspond to 5 points per wavelength (PPW) of the fundamental shear layer instability, in the transverse direction, and $10 \mathrm{PPW}$ in the streamwise direction. Analysis of the fourth-order compact scheme in use here, in several benchmark problems (Wilson 1996), has shown that roughly $6 \mathrm{PPW}$ are sufficient for accurate simulation. The compact scheme is inherently non-diffusive and non-dissipative, and with adequate resolution dispersive errors are minimized. 


\section{RESULTS AND DISCUSSION}

Figures 1 and 2 show computed instantaneous vorticity fields for cases (i) and (ii), respectively. In case (i), the flow remains symmetrical about the major and minor axes, just like the inlet mean flow and perturbation velocities, hence the flow has remained laminar and transition to turbulence did not occur in the 10 diameters computed. The phenomenon of axis switching is observed to be taking place near the end of the domain. The source of this is seen to be clearly in the complex vorticity field. It is most likely due to self-induction, since there is no streamwise vorticity at the inlet plane. It appears that the effect of the induced streamwise vorticity field is to pump fluid from the major axis to the minor axis. Now, since the flow remains laminar, this process is inviscid and is unrelated to turbulence-generated secondary flow as found in streamwise corners, the so-called secondary flow of Prandtl's second kind, (e.g., Demuren and Rodi 1984). The role of viscosity is to promote the eventual decay of this secondary motion. In case (ii), the vorticity field is even more complex, indicating the effects of the sub-harmonic component in inducing multiple vortex pairings. Near the end of the computed domain, some asymmetry can be discerned, indicating the beginning of the transition process. In this case, the phenomenon of axisswitching was not observed. It is therefore quite clear that the modes of instability waves present in the emerging boundary layer have strong effects on the evolution of the jet.

Time-averaged jet half-widths are shown for the two high Reynolds number cases, (iii) and (v) in Fig. 3. We see that in case (iii), with discrete two-mode forcing, no axis-switching occurred up to ten diameters downstream. This result is in agreement with that of case (ii), and is further confirmation of the thesis that the phenomenon of axis-switching is not controlled by the turbulence structure. On the other hand, in case (iv) axis-switching occurred at about 7 diameters. This inlet condition, with a broad-mode spectrum of perturbations is indicative of a jet evolving from a nozzle with turbulent boundary layers. Experimental data from similar conditions (Tsuchiya and Horikoshi 1986) suggest that axis-switching was observed at between 6 to 8 diameters. However, the presence of axis-switching should not be equated directly with increased entrainment of ambient fluid which is desired in several engineering applications. Integration of the entrained flow shows that more ambient fluid was entrained in case (iii) than in case (iv). 
All subsequent results focus on case (iv), which is closest to experiment of unforced jets. Figures 4 and 5 show instantaneous fields of the streamwise velocity, pressure, total vorticity, and the streamwise vorticity in the major and minor axis planes, respectively, after nine flow through times. Displayed numbers correspond to peak values for each variable, and negative values are drawn with dashed lines. The potential core of the jet extends to about 4 diameters, and significant streamwise vorticity commences from this location downstream. The latter quickly reached peak values by about 6 to 7 diameters, and subsequently decayed in strength further downstream. The location of axis cross-over corresponds roughly to the location of peak streamwise vorticity. Furthermore, although there are some pockets of positive instantaneous pressure, it is largely negative, thus fueling the entrainment of ambient fluid.

Figure 6 shows the balance of terms in the streamwise momentum equation, time-averaged over 1000 time steps and approximately 10 flow through times. We see that, by an overwhelming margin, the spatial convection is balanced by gradients of the resolved Reynolds stresses. By comparison, gradients of the SGS stresses are negligible, which would indicate the reliability of the LES approach for this problem even at the chosen Reynolds number of 75000 . The third most important term is the pressure gradient. In fact, near the edges, it is the dominant term which balances spatial convection, i.e., it acts as the source for the entrainment of ambient fluid. The symbols, in the figure, represent the lack of balance or drift from steady state, which is indicative of the adequacy of the sample size used in the time-averaging process. With no homogeneous direction in the present flow, the sample size seems to be adequate. Further time-averaged quantities of first and second moments, as well as budgets of the respective equations can be found in Wilson (1996).

\section{CONCLUDING REMARKS}

Numerical simulation of jets with rectangular cross-section have been performed using a third-order Runge-Kutta scheme for temporal integration and a fourth-order compact scheme for spatial discretization. Cases at low Reynolds numbers are direct simulations and those at high Reynolds number are large eddy simulations. 
The phenomenon of axis-switching is observed to be dependent on instability waves present in the inlet boundary layers, and could be induced in both laminar and turbulent jets. It is confirmed that this is based on self-induction of the vorticity field. The presence of a discrete sub-harmonic mode led to its suppression, but entrainment of ambient fluid was still enhanced.

Budget of terms in the mean streamwise momentum equation showed that spatial convection is balanced mostly by gradients of the Reynolds stresses, with pressure gradients making a minor contribution. However, near the edges, ambient fluid entrainment is fueled by the pressure gradients. Terms involving the SGS stresses and viscous diffusion are negligible.

\section{ACKNOWLEDGEMENT}

The authors wish to acknowledge many fruitful discussions with Dennis Bushnell. The simulations were performed on the Cray C-90 computer at NAS, NASA Ames Research Center, the use of which is gratefully acknowledged.

\section{REFERENCES}

Demuren, A. O. and Rodi, W. (1984) Calculation of Turbulence-Driven Secondary Motion in Non-Circular Ducts. J. Fluid Mech., 140, pp. 189-222.

Grinstein, F. F. (1995) Self-Induced Vortex Ring Dynamics in Subsonic Rectangular Jets. Phys. Fluid., 7, pp. 2519-2521.

Lele, S. K. (1992) Compact Finite Difference Schemes with Spectral-Like Resolution. J. Comp. Phys. 103, pp. 16-42.

Lowery, S. L. and Reynolds, W. C. (1986) Numerical Simulation of a Spatially-Developing Mixing Layer. Report TF-26, Mech. Engr. Dept., Stanford Univ.

Miller, R. S., Madnia, C. K. and Givi, P. (1995) Numerical Simulation of Non-Circular Jets. Comp. Fluid., 24, pp. 1-25. 
Quinn, W. R. (1989) On Mixing in an Elliptic Turbulent Free Jet. Phys. Fluid. A, Vol. 1, No. 10 , pp. 1716-1722.

Quinn, W. R. (1994) Experimental Study of a Turbulent Free Jet Issuing from a Small-AspectRatio Sharp-Edged Rectangular Slot. Proc. 2nd Int. Conf. Expt. Fluid Mech., Turin, Italy.

Tsuchiya, Y. and Horikoshi, C. (1986) On the Spread of Rectangular Jets. Expt. Fluid., 4, pp. 197-204.

Tsuchiya, Y., Horikoshi, C., Sato, T. and Takahashi, M. (1986) A Study on the Spread of Rectangular Jets. JSME Int. J., II, 32, pp. 11-18.

Williamson, J. (1980) Low Storage Runge-Kutta Schemes. J. Comp. Phys., 35, pp. 48-56.

Wilson, R. V. (1993) Numerical Simulation of Two-Dimensional, Spatially Developing Mixing Layers. Masters Thesis, Old Dominion University.

Wilson, R. V. (1996) Numerical Simulation of Complex Three-Dimensional Turbulent Free Jets. Ph.D. Thesis, Old Dominion University.

Wilson, R. V. and Demuren, A. O. (1996) Two-Dimensional Spatially-Developing Mixing Layers, Numerical Heat Transfer, A., 29, pp. 485-509. 
(a)

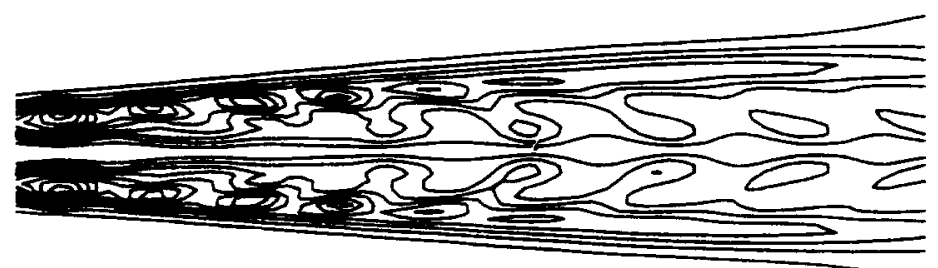

(b)

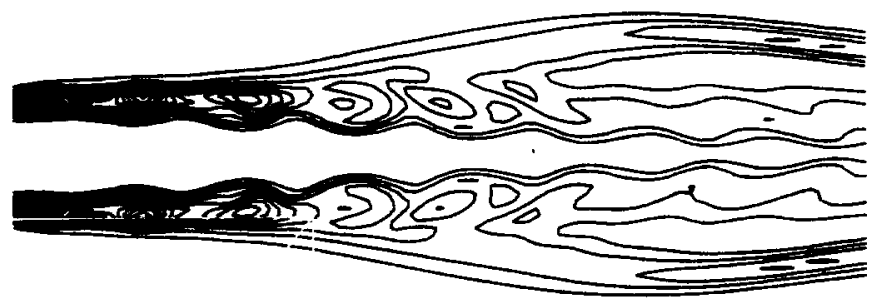

(c)

(d)

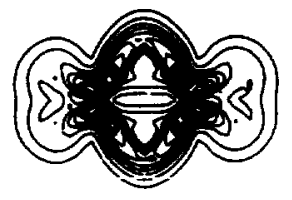

(e)

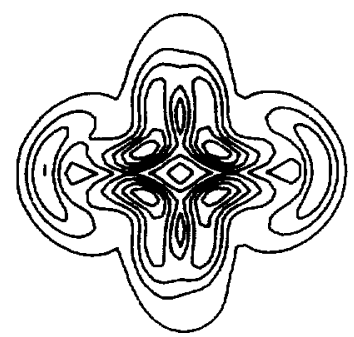

(f)

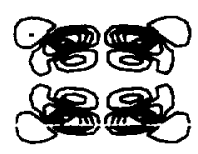

(g)

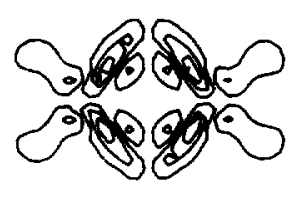

(h)

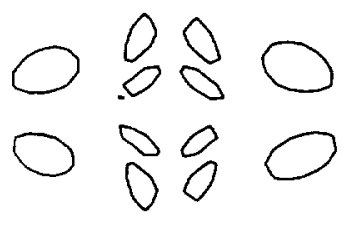

Figure 1. Contours of vorticity magnitude, (a) - (e) and streamwise vorticity, (f) - (h) for case (i) at $t=2$ flow through times, for fundamental forcing function. (a) minor axis plane, $z / D_{e}=$ 0 , (b) major axis plane, $y / D_{e}=0$, (c) cross flow plane, $x / D_{e}=0$, (d) $x / D_{e}=5$, (e) $x / D_{e}=$ 10 , (f) $x / D_{e}=2.5,(\mathrm{~g}) x / D_{e}=5$, and (h) $x / D_{e}=10$. 
(a)

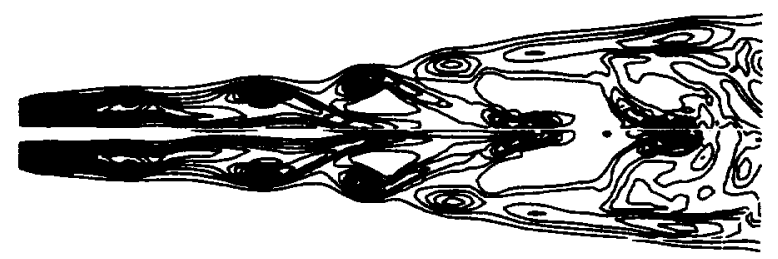

(b)

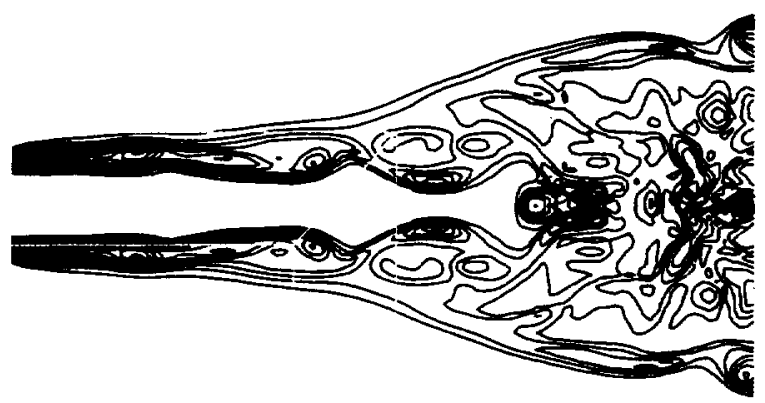

(c)

(f)

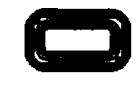

(d)

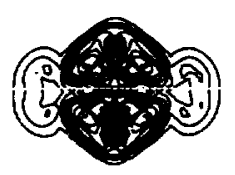

(e)

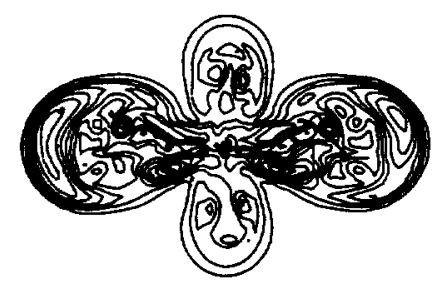

$0_{2} 0$

00

(g)

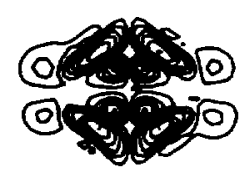

(h)

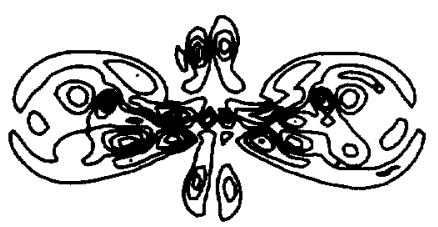

Figure 2. Contours of vorticity magnitude, (a) - (e) and streamwise vorticity, (f) - (h) for case (ii) at $t=2$ flow through times, for fundamental and first subharmonic forcing function. (a) minor axis plane, $z D_{e}=0$, (b) major axis plane, $y / D_{e}=0$, (c) cross flow plane, $x / D_{e}=0$, (d) $x / D_{e}=5$, (e) $x / D_{e}=10$, (f) $x / D_{e}=2.5$, (g) $x / D_{e}=5$, and (h) $x / D_{e}=10$. 

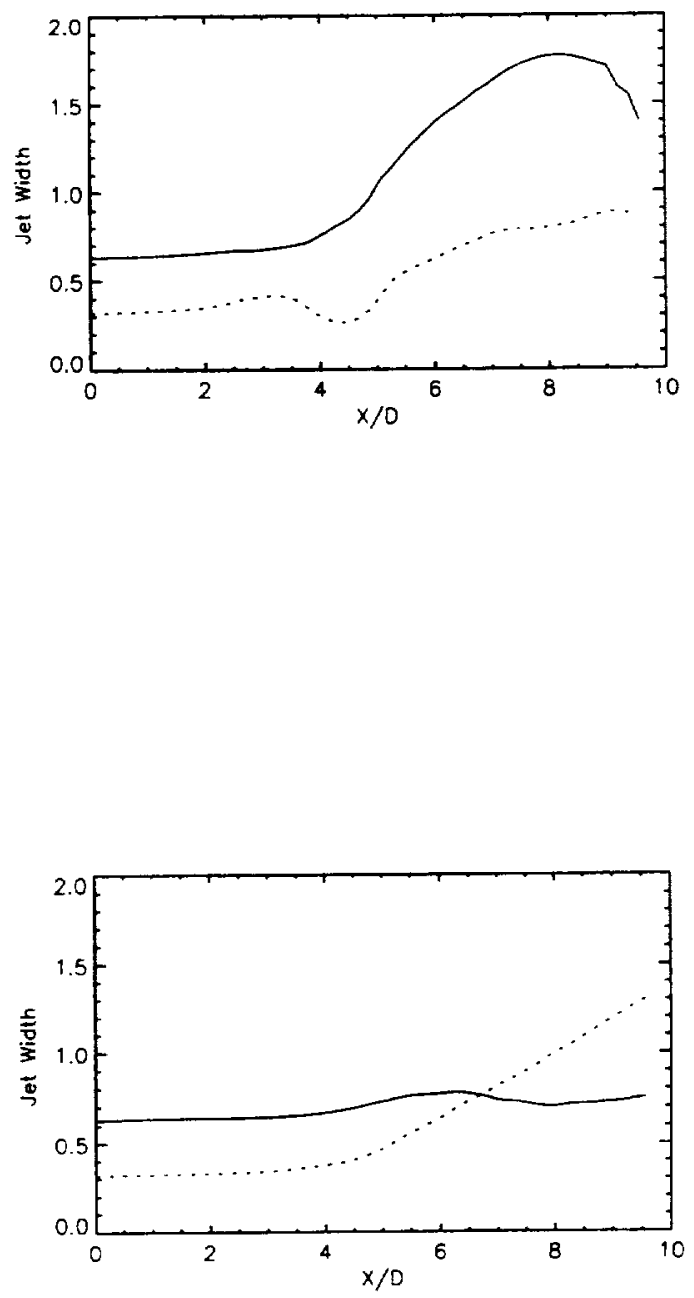

Figure 3. Jet half-widths for (a) case (iii), (b) case (iv) (solid - major axis plane, dashed - minor axis plane) 

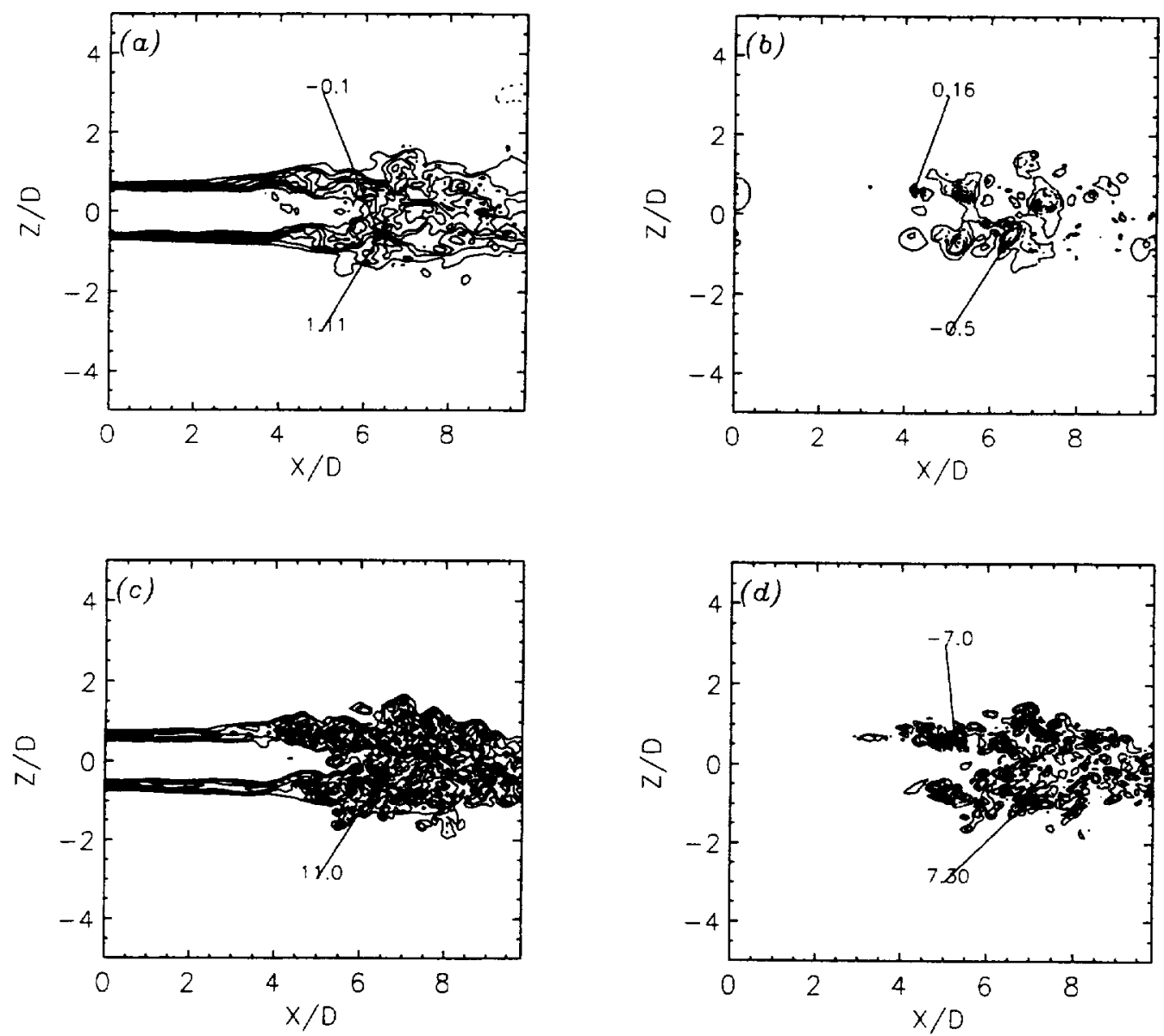

Figure 4. Instantaneous contours of dimensionless (a) streamwise velocity, (b) pressure, (c) total vorticity, (d) streamwise vorticity along major axis plane at $t=9$ flow through times, for case (iv). (Numbers represent peak values). 

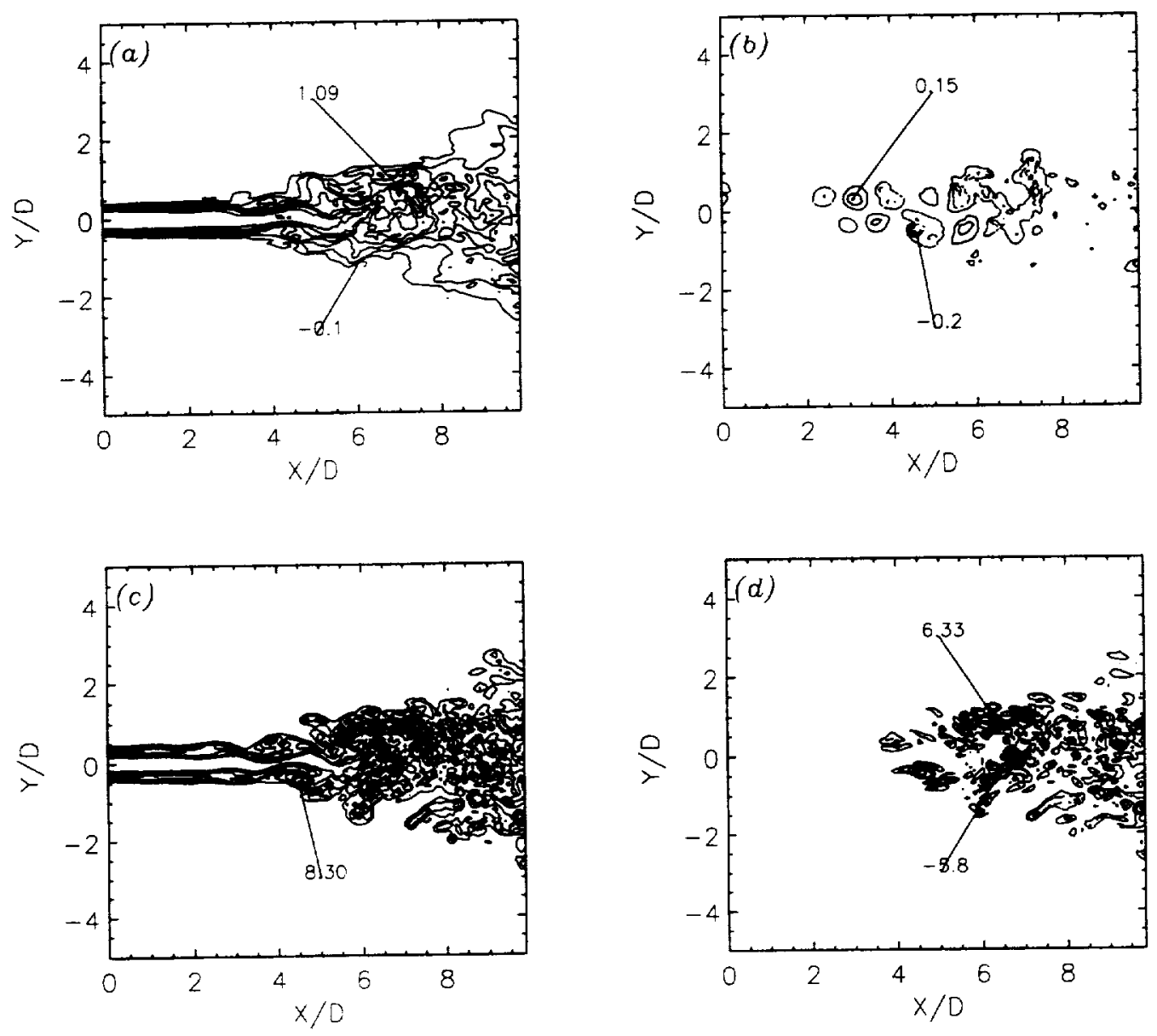

Figure 5. Instantaneous contours of dimensionless (a) streamwise velocity, (b) pressure, (c) total vorticity, (d) streamwise vorticity along minor axis plane, at $t=9$ flow through times, for case (iv). (Numbers represent peak values). 

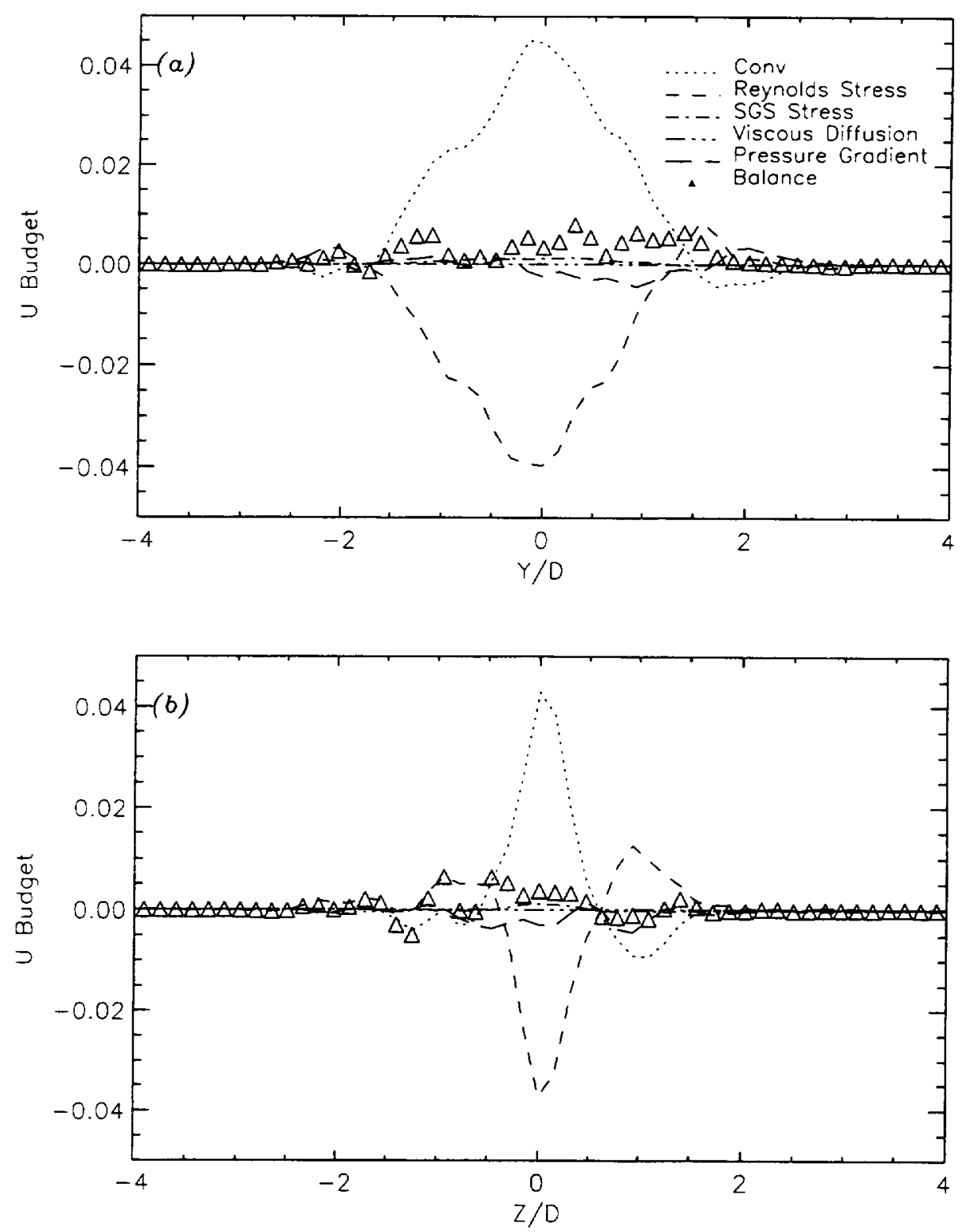

Figure 6. Balance of terms in the mean streamwise momentum equation, for case (iv) at $x / D_{e}=$ 9.4. (a) minor axis; (b) major axis. 
Public reporting burden for this collection of information is estimated to average 1 hour per response, including the time for reviewing instructions, searching existing data sources. gathering and maintaining the data needed, and completing and reviewing the collection of information. Send comments regarding this burden estions and Reports, 1215 Jefferson Davis Highway, Suite 1204. Arlington, VA 22202-4302, and to the Office of Management and Budget, Paperwork Reduction Project (0704-0188), Washington, DC 20503.

\begin{tabular}{l|l|l}
\hline 1. AGENCY USE ONLY(Leave blank) & 2. REPORT DATE & 3. REPORT TYPE AND DATES COVERED
\end{tabular}

1. AGENCY USE ONLY(Leave blank) $\begin{gathered}\text { 2. REPORT DATE } \\ \text { January } 1997\end{gathered} \quad \begin{gathered}\text { 3. REPORT TYPE AND Dh } \\ \text { Contractor Report }\end{gathered}$

4. TITLE AND SUBTITLE

5. FUNDING NUMBERS

Numerical simulation of turbulent jets with rectangular cross-section

C NAS1-19480

WU 505-90-52-01

6. AUTHOR(S)

Robert V. Wilson

Ayodeji O. Demuren

7. PERforming ORganization nAME(S) AND ADDRESS(ES)

Institute for Computer Applications in Science and Engineering

Mail Stop 403, NASA Langley Research Center

Hampton, VA 23681-0001

8. PERFORMING ORGANIZATION

REPORT NUMBER

ICASE Report No. $97-1$

9. SPONSORING/MONITORING AGENCY NAME(S) AND ADDRESS(ES)

National Aeronautics and Space Administration

Langley Research Center

Hampton, VA 23681-0001

10. SPONSORING/MONITORING

AGENCY REPORT NUMBER

NASA CR-201642

ICASE Report No. 97-1

\section{SUPPLEMENTARY NOTES}

Langley Technical Monitor: Dennis M. Bushnell

Final Report

To be submitted to the ASME Journal of Fluids Engineering.

\begin{tabular}{l|l|l}
\hline 12a. DISTRIBUTION/AVAILABILITY STATEMENT & 12b. DISTRIBUTION CODE
\end{tabular}

Unclassified-Unlimited

Subject Category 34

13. ABSTRACT (Maximum 200 words)

Three-dimensional turbulent jets with rectangular cross-section are simulated with a finite-difference numerical method. The full Navier-Stokes equations are solved at low Reynolds numbers, whereas at the high Reynolds numbers filtered forms of the equations are solved along with a subgrid scale model to approximate effects of the unresolved scales. A 2-N storage, third-order Runge-Kutta scheme is used for temporal discretization and a fourthorder compact scheme is used for spatial discretization. Computations are performed for different inlet conditions which represent different types of jet forcing. The phenomenon of axis-switching is observed, and it is confirmed that this is based on self-induction of the vorticity field. Budgets of the mean streamwise velocity show that convection is balanced by gradients of the Reynolds stresses and the pressure.

\begin{tabular}{|c|c|c|c|}
\hline \multicolumn{3}{|c|}{$\begin{array}{l}\text { 14. SUBJECT TERMS } \\
\text { Rectangular Jets; Large Eddy Simulation; Direct Simulation; Turbulence }\end{array}$} & $\begin{array}{c}\text { 15. NUMBER OF PAGES } \\
19 \\
\end{array}$ \\
\hline $\begin{array}{l}\text { 17. SECURITY CLASSIFICATION } \\
\text { OF REPORT } \\
\text { Unclassified }\end{array}$ & $\begin{array}{l}\text { 18. SECURITY CLASSIFICATION } \\
\text { OF THIS PAGE } \\
\text { Unclassified }\end{array}$ & $\begin{array}{l}\text { 19. SECURITY CLASSIFICATION } \\
\text { OF ABSTRACT }\end{array}$ & $\begin{array}{l}\text { 20. LIMITATION } \\
\text { OF ABSTRACT }\end{array}$ \\
\hline
\end{tabular}

